\title{
Position sensitive twin ionization chamber for nuclear fission investigations
}

\author{
Shakir Zeynalov*, Pavel Sedyshev ${ }^{\dagger}$, Olga Sidorova $^{\ddagger}$ and Valery Shvetsov ${ }^{\S}$ \\ Frank Laboratory of Neutron Physics, Joint Institute for Nuclear Research, Joliot-Curie 6 \\ Dubna, Moscow region 141980, Russian Federation \\ *zeinal@nf.jinr.ru \\ †sedyshev@nf.jinr.ru \\ siolia@mail.ru \\ sshv@nf.jinr.ru \\ Leonid Svetov \\ Dubna International University of Nature, Society and Man \\ Dubna, Moscow region 141980, Russian Federation \\ lenzo@list.ru
}

Published 10 July 2018

\begin{abstract}
In this article, we report the recent achievements in the design of a back-to-back twin ionization chamber (TIC) for fission fragment (FF) mass, kinetic energy, and orientation. Correlated FF kinetic energies, their masses, and the angle of the fission axes in 3D Cartesian coordinates can be determined from analysis of the heights and shapes of the pulses induced by the FFs on the anodes of the TIC. These anodes were designed to consist of isolated $\Delta$-shaped strips connected to nodes of a chain filter made of serially-connected two-port networks. The double charge division method was implemented by digitizing four waveforms at the endpoints of the chain filters. It was shown that the fission fragments emission point on the target plane may be determined using the measured data. A position-sensitive neutron-induced fission detector for neutron imaging applications with both thermal and low energy neutrons was found as another possible implementation of the designed TIC. Preliminary measurements with thermal neutron-induced fission were done with RC chain filters and the results are reported here.
\end{abstract}

Keywords: Ionization chambers; fission; thermal neutrons.

PACS numbers: 29.30.Hs, 29.40.Cs, 29.40.Mc, 29.85.Fj, 25.85.Ca

\section{Introduction}

The nuclear fission model and prompt fission neutron (PFN) emission was first developed by Niels Bohr and John Wheeler. In their work, nuclei were perceived as a drop of charged liquid that a surface could constantly distort in competition between attractive nuclear and repulsive Coulomb forces. Infrequently, a large distortion brought the nucleus into a configuration where repulsion could not be compensated by the nuclear

This is an Open Access article published by World Scientific Publishing Company. It is distributed under the terms of the Creative Commons Attribution 4.0 (CC-BY) License. Further distribution of this work is permitted, provided the original work is properly cited. 
force and the system split, sometimes after neutron emission. In this case, neutrons called scission neutrons (to distinguish them from PFNs) were emitted from the fully accelerated fission fragments (FF). The configuration of this nuclear shape just before the split can be monitored experimentally by measuring the FF kinetic energy release along with the PFN velocity, and the angle between the fission axis and the PFN measured in a single fission event. In a new experimental approach developed in Ref. 1, the authors investigated PFN emission in spontaneous fission of ${ }^{252} \mathrm{Cf}$ using a Frisch-grid twin ionization chamber (TIC) for FF kinetic energies and a PFN emission angle along with PFN velocity measurement with the help of a liquid scintillator (NE213 or equivalent liquid) based neutron detector (ND). The authors demonstrated the power and high capacity of the new approach, which was further increased by replacing the traditional analog electronics by modern digital pulse processing (DPP) as described in Ref. 2 . The PFN detection efficiency of the method was limited by the use of only two fast neutron detectors-located along the TIC axis-because the FF angle in the TIC could only be measured with respect to a certain axis. When the PFN detector was located along this axis, the measured angle was the same as between FF and PFN emissions. Therefore, the next modification of the method was intended to measure correlated FF angles with respect to three axes of a 3D Cartesian coordinate frame in an event-by-event basis. If the PFN detector's location in the same Cartesian frame was fixed and well known, then the angle between FF and PFN emission could be evaluated for each fission event. It should be noted that two correlated FFs were emitted along the straight line called the fission axis for the considered fission event. That means that for each fission event, the coordinates of two points on the fission axis could be measured. ${ }^{2,3}$ This provided the possibility to evaluate the two-dimensional (2D) coordinates of the crossing point of the fission target plane with a fission axis-neutron capture location. The modification was expected to improve the quality of experiments with targets like ${ }^{239} \mathrm{Pu},{ }^{235} \mathrm{U}$, and ${ }^{237} \mathrm{~Np}$ in resonance neutron-induced fission. In addition, the slight modification of the developed $2 \mathrm{D}$ coordinate readout principle could became a very competitive solution for neutron imaging, tomography, and similar applications.

\section{Shockley-Ramo Theorem and Charge Division}

Using the Shockley-Ramo theorem, ${ }^{4}$ a calculation of the so-called weighting potential in the three dimensional (3D) Cartesian coordinate system was performed. According to the theorem condition, the weighting potential in the TIC volume was calculated for one $\Delta$ electrode potential raised to 1, leaving other electrodes in Fig. 1 grounded. If the strips were operated at positive potential relative to the cathode surface, then ionization electrons would be attracted along the real field lines (calculated for a homogeneous electric field between anode and cathode). From the calculated weighting potential F(x,y, $\mathrm{z})$, the slices $\mathrm{F}(\mathrm{x}, \mathrm{y}=$ const, $\mathrm{z}$ ) were derived and plotted in Fig. 2 to demonstrate the charge division along the $\mathrm{Y}$ coordinate of the anode plane. The full scale of $\mathrm{X}, \mathrm{Y}$, and $\mathrm{Z}$ coordinates is 400,300 , and 300 units respectively. It should be noted that the weighting potential is almost zero everywhere except in the close vicinity of the anode plane. If the 
cathode-anode separation is chosen to be large enough to keep the FFs from stopping too close to the anode plane, then there is no need for a Frisch-grid as a screening electrode. The linear behaviour of the charge division between correlated $\Delta$-electrodes was concluded from the Y-dependence of the weighting potentials.

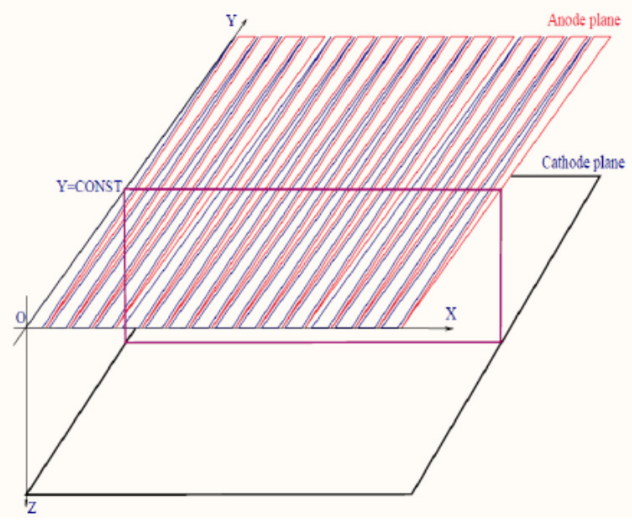

Fig. 1. The arrangement of the electrodes for one of the TICs.
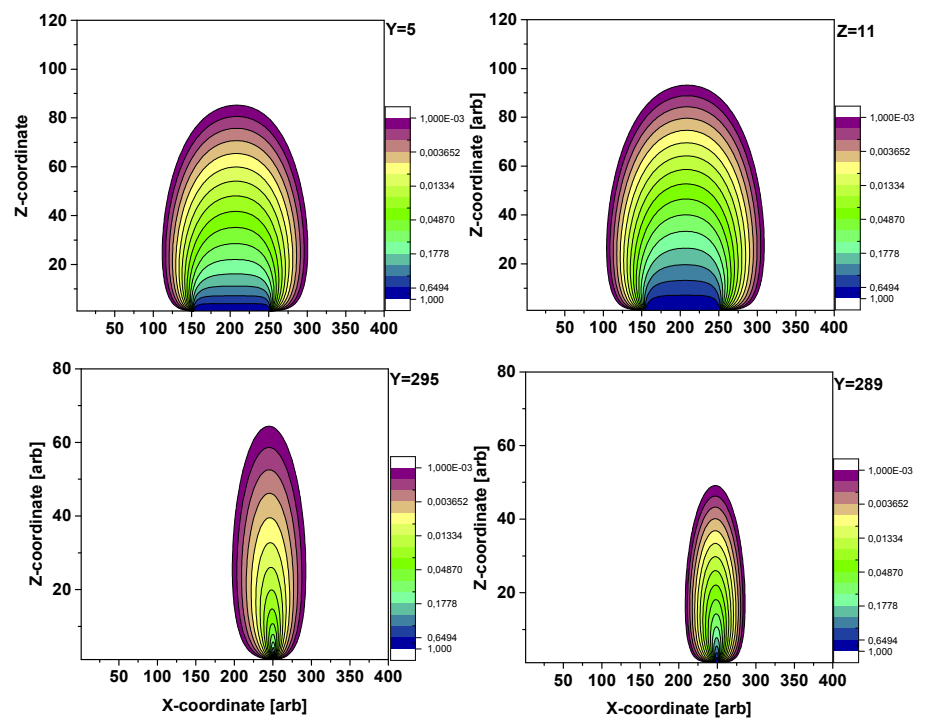

Fig. 2. Weighting potential $F(x, y=$ const, $z$ ) shown for $\mathrm{Y}=5$ and $\mathrm{Y}=11$ along with $\mathrm{Y}=295$ and $\mathrm{Y}=289$ for complementary $\Delta$-electrodes.

This fact was used to evaluate the Y-coordinate value from the induced signals on the anode. The charge induced on the $\Delta$-electrode was calculated according to ShockleyRamo theorem as

$$
Q=q \cdot \Delta F,
$$


where $\Delta F$ is the difference of weighting potentials between the point of charged particle $q$ origin and the point where the charged particle was collected at the anode surface, and $Q$ is the induced charge. The FFs create ionization electrons along their deceleration path in the TIC working gas. In the description of pulse formation in the TIC, the charge "center of gravity" is considered to be a good measure of the FF coordinate. Therefore, in this paper all calculations of FF coordinates refer to that value, which is calculated according to the formula

$$
X=\frac{1}{Q_{f}} \int_{0}^{\infty} x \cdot \rho(x) \cdot d x, \quad Q_{f}=\int_{0}^{\infty} \rho(x) \cdot d x,
$$

where the integration is done along the FF deceleration path, $\rho(x)$ is the density distribution of ionization electrons along the FF deceleration path, and $Q_{f}$ is the full charge of ionization electrons.

\section{Charge Division on the Chain Filter}

The position information along the X-coordinate was obtained by two methods: (1) the charge attenuation and (2) the pulse delay. Both methods were based on the charge splitting at the node of the chain filter. The chain filter was formed by serially-connected two-port networks created by the resistance $R$, inductance $L$ and capacitance $C$ of the $\Delta$ electrodes. The circuit diagram of the chain filter made of passive electrical components is depicted in Fig. 3. If the chain filter is terminated by a wave impedance $Z_{\mathrm{W}}$, as shown in the figure, then the charge would split into two equal portions between the two ends of the filter.

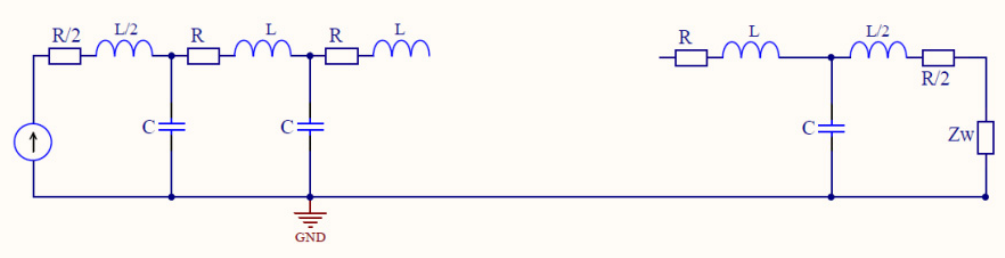

Fig. 3. Circuit diagram of the chain filter.

Operational calculus is a conventional method to study the signal propagation along serially connected two-port networks. If the resistance can be neglected, then Eq. (3) represents the Laplace transformation of the unit step signal after passing through $\mathrm{m}$ twoport networks.

$$
i_{m}(t) \supset \sqrt{\frac{C}{L}} \frac{1}{p} \frac{1}{\sqrt{\left(\frac{b}{p}\right)^{2}+1} \cdot\left[\frac{b}{p}+\sqrt{\left(\frac{b}{p}\right)^{2}+1}\right]^{2 m}}, \quad \mathrm{~b}=\frac{1}{2 \sqrt{\mathrm{LC}}} .
$$


The inverse Laplace transformation can be found using transform tables: ${ }^{5}$

$$
i_{m}^{i n d}(t)=\sqrt{\frac{C}{L}} \int_{0}^{\infty} J_{0}(2 \sqrt{b t \tau}) J_{2 m}(\tau) d \tau
$$

Equation (4) describes propagation of the unit step signal over a chain filter made of inductance and capacitance (the resistance is very small). For the case where the resistance cannot be neglected, the result is as follows:

$$
i_{m}^{r e s}(t)=\sqrt{\frac{C}{L}} \int_{0}^{t} \exp \left(-\frac{R}{L} \cdot t\right) J_{2 m}\left(\frac{2 \cdot t}{\sqrt{L C}}\right) d\left(\frac{2 \cdot t}{\sqrt{L C}}\right) .
$$

Equation (5) describes propagation of the unit step signal over a chain filter made of resistance and capacitance (inductance very small). The wave impedance and the speed of signal propagation are given by the following formulas for both cases considered above:

$$
Z_{w}=\sqrt{\frac{(R+p l)^{2}}{4}+\frac{R+p L}{p C}}, \quad \frac{m}{t}=\frac{1}{\sqrt{L C}} .
$$

It should be noted that in the case of a resistive chain filter, the pulse height of the unit step signal is attenuated exponentially as it passes through the chain filter.

\section{Numerical Simulations}

The dependence of output pulse height on the sequential number of two-port networks that the signal has passed through in the 16-network chain filter is plotted in Fig. 4. The calculation was done using a digital RC-filter to represent the two-port network. The attenuation of the unit step signal height by a factor of $\exp (-\mathrm{m} / \mathrm{T})$ on passing sequentially through $\mathrm{m}$ two-port networks was calculated and plotted in Fig. 5 (left), and the T value was found by fitting the $\exp (-\mathrm{m} / \mathrm{T})$ function. The $\mathrm{T}$ parameter can be used to correct the attenuation of total charge induced on the strips.
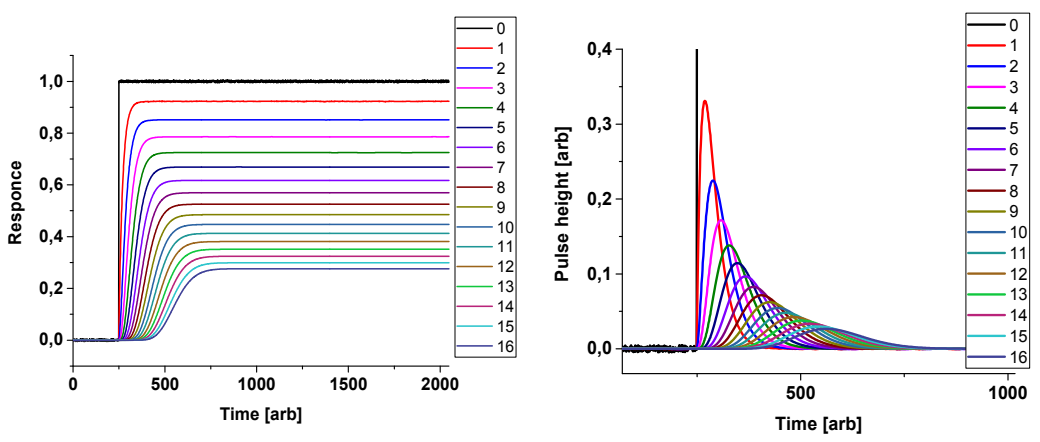

Fig. 4. Unit step signal attenuation after passing through the $\mathrm{m}^{\text {th }}$ two-port network (left), and the pulses obtained after differentiating the unit step signals (right). 

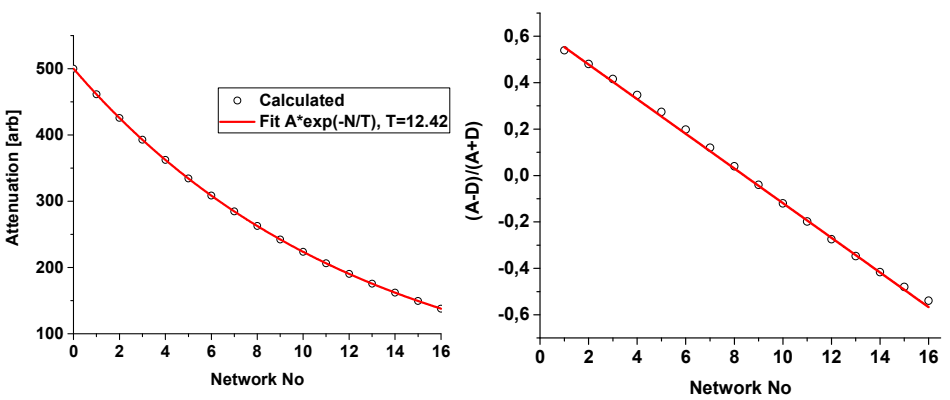

Fig. 5. Attenuation of unit step signal height after passing two-port network number $\mathrm{N}$ (left) and position evaluated from simulated data (right).

The coordinate $m$ of the charge "center of gravity" was evaluated from the simulated pulses using the following formula:

$$
m=\frac{A-B}{A+B},
$$

where $A$ and $B$ are the pulse heights calculated for the two-port network with sequential number $m$ and 16- $m$ respectively. The result demonstrates almost perfect linear dependence on the network number (Fig. 5, right). Another way to determine the coordinate $m$ was connected to measurement of the delay between the unit step signal and its response at the ends of the two-port networks as demonstrated in the plot of Fig. 5 (right).

\section{Experimental Measurements}

Experiments were performed with the ionization chamber with a rectangular-shaped anode made of 16 strips, each strip consisting of two $\Delta$-electrodes as shown in Fig. 6. Each $\Delta$-electrode was connected to the nodes of the sequentially connected resistors. These resistors connected with $\Delta$-electrode capacitance and created a two-port network. The ending resistors were connected to the input of a charge-sensitive preamplifier. Output signals of the preamplifiers were digitized by four synchronous waveform digitizers (WFD) with sampling frequency of 250 megahertz $(\mathrm{MHz})$ and pulse height resolution of 8 bits. The target was made of ${ }^{235} \mathrm{UF}_{6}$ and deposited on the surface of a thick Al foil. The target thickness was $\sim 500 \mu \mathrm{G} / \mathrm{cm}^{2}$, and it was mounted on the center of a circular cathode with diameter $180 \mathrm{~mm}$. The distance between the cathode and the anode was $\sim 45 \mathrm{~mm}$. The working gas of the ionization chamber was a standard P10 mixture $\left(90 \% \mathrm{Ar}+10 \% \mathrm{CH}_{4}\right)$. The chamber was operated at a constant gas flow of $\sim 35 \mathrm{ml} / \mathrm{min}$ with a pressure of 1.07 bars. FFs were produced by a thermal neutron flux of $\sim 10^{4} \mathrm{n} / \mathrm{s}$. The capture of thermal neutrons by ${ }^{235} \mathrm{U}$ nuclei caused the fission, producing two correlated FFs, one of which was decelerated in the working gas of the ionization chamber. Ionization electrons created by the FF drifted along the electric field lines 
toward the anode, inducing the signals on the $\Delta$-electrodes. After amplification, the signals were recorded by the four WFDs and stored in PC memory for further offline data analysis. There were four waveforms recorded for each fission event, which were used for FF charge "center of gravity" evaluation. The organization of the measurement apparatus provided the possibility to make measurements of each coordinate in two different independent ways. This was used to evaluate the precision of the coordinate measurement as demonstrated in Fig. 7.

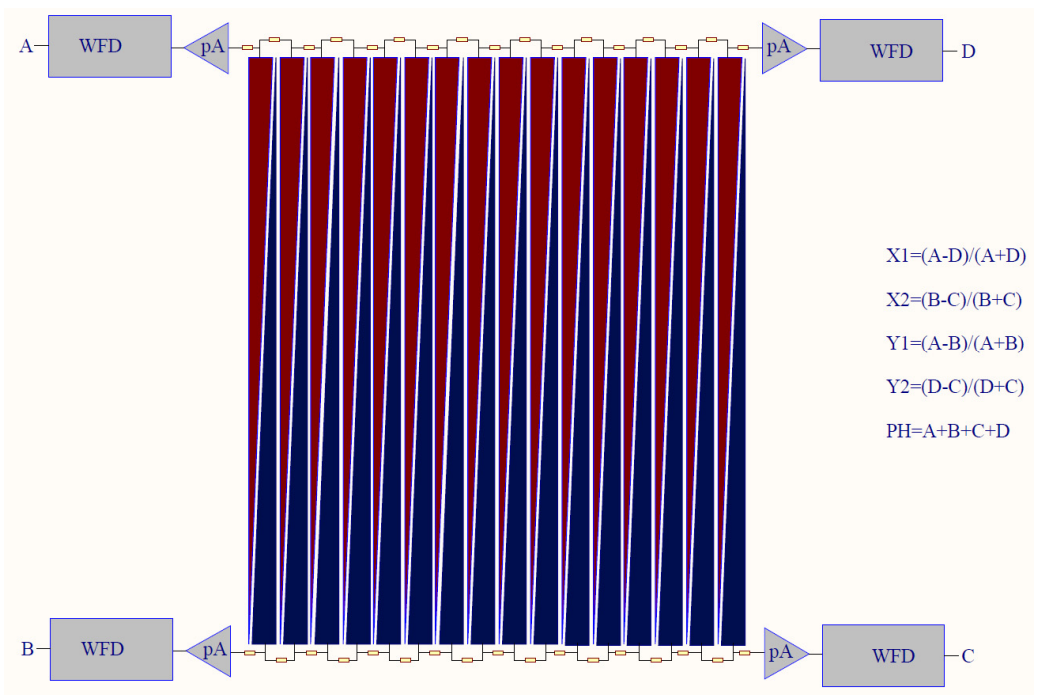

Fig. 6. Sketch of data readout system used in the experiment.
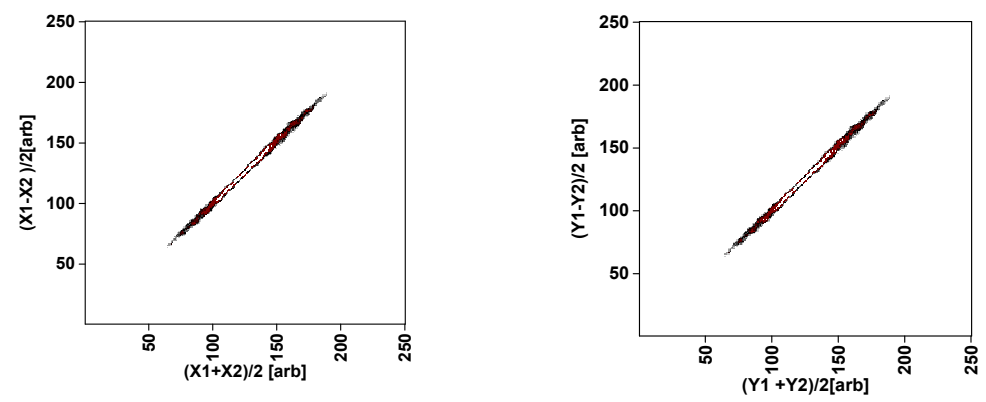

Fig. 7. Two dimensional plots demonstrating the scattering of measured data with respect to average values for $\mathrm{X}$ coordinates (left) and $\mathrm{Y}$ coordinates (right) in the anode plain. 
For example, the coordinate $X$ was measured as:

$$
X_{1}=\frac{A-D}{A+D} \text { and } X_{2}=\frac{B-C}{B+C}
$$

where A, B, C, and D are the pulse heights obtained from the correlated waveforms and recorded for the same fission event. These measured coordinates were used to make a $2 \mathrm{D}$ plot $P(x, y)$ in coordinates $x, y$ calculated for each fission event using the formulas:

$$
x=\left(X_{1}+X_{2}\right) / 2, y=\left(X_{1}-X_{2}\right) / 2 .
$$

The plot of $P(x, y)$ is shown above in Fig. 7 (left) and demonstrates scattering of the points due to random errors. The function $G(x, y)$ was constructed in the way similar to $P(x, y)$ using the following formulas:

$$
Y_{1}=\frac{A-B}{A+B} \text { and } Y_{2}=\frac{D-C}{D+C} .
$$

From the values of $Y_{1}, Y_{2}$, coordinates of the 2D function $G(x, y)$ were constructed using the following formulas:

$$
x=\left(Y_{1}+Y_{2}\right) / 2, y=\left(Y_{1}-Y_{2}\right) / 2 .
$$

The plot of $G(x, y)$ is shown above in Fig. 7 (right). The measured functions $P(x, y)$ and $G(x, y)$ were used for precision evaluation of $x, y$ coordinate measurements. It was done by integrating functions $P(x, y)$ and $G(x, y)$ over variable $x$. The results are presented in Fig. 8 (left).
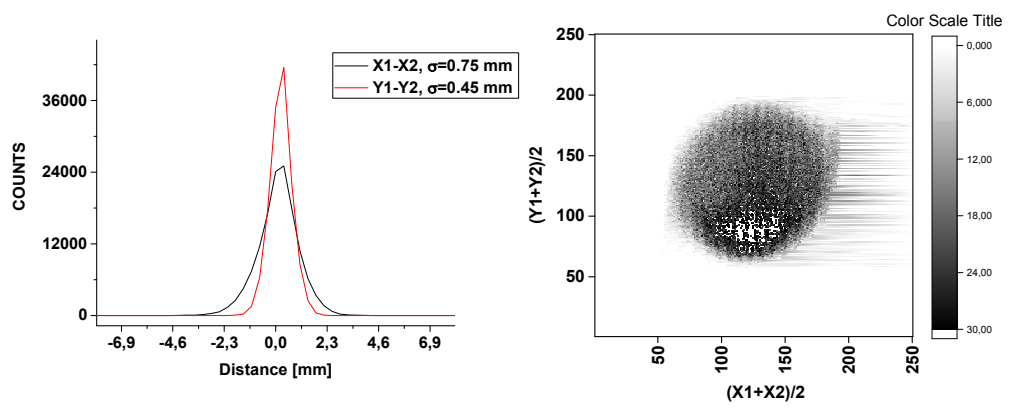

Fig. 8. One dimensional plot demonstrating precision of position of charge "gravity center" measurement (left); image of the ${ }^{235} \mathrm{U}$ target (right).

The image of the ${ }^{235} \mathrm{U}$ target measured in this experiment is shown in Fig. 8 (right). It should be noted that measurements were done with one of the TIC chambers due to the target backing being thick enough to absorb the complementary FF. That is why the fissile nucleus position could not be measured directly in the experiment performed. The kinetic energy release of the FF was analyzed using all four waveforms, recorded on an 
event by event basis. These waveforms first were corrected for attenuation in the chain filter as was described above. The corrected waveforms were unfolded to a single signal taking into account that sampling of the four signals actually was made sequentially with $1 \mathrm{GHz}$ frequency. That means that first signal sampled was at the first rising edge of the $1 \mathrm{GHz}$ oscillator, the second signal at the next edge, and so on. The unfolded signal was used for evaluation of the Z-coordinate of the FF charge "center of gravity" and the angles between the FF and Cartesian frame axes as it was described in Refs. 2, 3, and 7. Using the coordinates, kinetic energy, and angle measured for one of the FF, similar parameters for the correlated FFs were evaluated neglecting the target thickness. The correlated FF kinetic energy was calculated using the total kinetic energy value for the ${ }^{235} \mathrm{U}\left(\mathrm{n}_{\mathrm{th}}, \mathrm{f}\right)$ reaction known from the literature. ${ }^{6}$ The location of the point on the target plane was then evaluated as the crossing point of the straight line drawn between two "center of gravity" points of two correlated FFs. The evaluated accuracy of the coordinates was found to be better than $0.2 \mathrm{~mm}$.

\section{Conclusions}

The theoretical and experimental investigation of signal propagation through a chain filter made of serially-connected, two-port networks was performed with the objective of position sensitive ionization chamber design for PFN emission investigations with fast neutron detectors at arbitrary locations. The relationship between 2D Cartesian coordinate $(\mathrm{X}, \mathrm{Y})$ information and the response of the chain filter was found and investigated by digital simulation. It was shown that coordinate information can be obtained by both the double charge division and time delay method. Implementing both methods provided better accuracy in coordinate measurement. The dependence of pulse height data on coordinates was investigated for a resistive chain filter. A procedure for pulse height data correction was developed. Measurements of neutron imaging with a U235 target were done to demonstrate the quality of the double charge division method for the position sensitive ionization chamber. Good position resolution was demonstrated: $0.7 \mathrm{~mm}$ for $\mathrm{X}$ and $0.5 \mathrm{~mm}$ for $\mathrm{Y}$ coordinates. A new design for a He-3 imaging proportional chamber with the double charge division method could use the method to improve the coordinate resolution in comparison with a double delay line readout system. Digitization electronics were implemented for the data acquisition system, and the data analysis software was developed and tested in experiments. The data analysis was done using DPP algorithms developed by some of the present authors ${ }^{7}$ as the recursive procedures.

\section{References}

1. C. Budtz-Jorgensen, H.-H. Knitter, Ch. Straede, F.-J. Hambsch and R. Vogt, Nucl. Instr. Meth. A 258, 209 (1987).

2. Sh. Zeynalov, O. Zeynalova, M.A. Nazarenko, F.-J. Hambsch and S. Oberstedt, AIP. Conf. Proc. 1487, 405 (2012), doi: 10.1063/1.4758984. 
3. A. Gook, W. Geerts, F.-J. Hambsch, S. Oberstedt, M. Vidali and Sh. Zeynalov, Nucl. Instr. Meth. A 830, 366 (2016).

4. G.F. Knoll, Radiation Detection and Measurements (Wiley, New York, 2000), p. 789.

5. G.A. Korn and T.M. Korn, Mathematical Handbook for Scientists and Engineers (McGrawHill Book Company, New York, 1968 edition), p. 926.

6. C. Wagemans (ed.), The Nuclear Fission Process (CRC Press, Boca Raton, 1991), p. 323.

7. L. Svetov, O. Sidorova and Sh. Zeinalov, in Proc. XXIV International Seminar on Interaction of Neutrons with Nuclei, JINR E3-2017-8 (Dubna, 2017), p. 430. 